\title{
HELLS serves as a poor prognostic biomarker and its downregulation reserves the malignant phenotype in pancreatic cancer
}

Feng-Jiao Wang ${ }^{1,2+}$, Yan-Hua Jing ${ }^{1,2+}$, Chien-Shan Cheng ${ }^{1,2}$, Zhang-Qi Cao ${ }^{1,2}$, Ju-Ying Jiao ${ }^{1,2}$ and Zhen Chen ${ }^{1,2^{*}}$

\begin{abstract}
Background: SMARCAs, belonged to SWI/SNF2 subfamilies, are critical to cellular processes due to their modulation of chromatin remodeling processes. Although SMARCAs are implicated in the tumor progression of various cancer types, our understanding of how those members affect pancreatic carcinogenesis is quite limited and improving this requires bioinformatics analysis and biology approaches.
\end{abstract}

Methods: To address this issue, we investigated the transcriptional and survival data of SMARCAs in patients with pancreatic cancer using ONCOMINE, GEPIA, Human Protein Atlas, and Kaplan-Meier plotter. We further verified the effect of significant biomarker on pancreatic cancer in vitro through functional experiment.

Results: The Kaplan-Meier curve and log-rank test analyses showed a positive correlation between SMARCA1/2/3/ SMARCAD1 and patients' overall survival (OS). On the other hand, mRNA expression of SMARCA6 (also known as HELLS) showed a negative correlation with OS. Meanwhile, no significant correlation was found between SMARCA4/5/SMARCAL1 and tumor stages and OS. The knockdown of HELLS impaired the colony formation ability, and inhibited pancreatic cancer cell proliferation by arresting cells at $S$ phase.

Conclusions: Data mining analysis and cell function research demonstrated that HELLS played oncogenic roles in the development and progression of pancreatic cancer, and serve as a poor prognostic biomarker for pancreatic cancer. Our work laid a foundation for further clinical applications of HELLS in pancreatic cancer.

Keywords: SMARCA, HELLS, Pancreatic cancer, Prognostic value, Data mining analysis

\section{Introduction}

The switch/sucrose nonfermenting complex (SWI/SNF) is an ATPase-dependent multisubunit complex modulating gene expression involved in chromatin remodeling and transcriptional regulation [1]. The whole-exome sequencing data, including 18 neoplastic entities from 24 published studies, showed widespread SWI/ SNF mutations among diverse human cancers (20\%),

\footnotetext{
${ }^{*}$ Correspondence: zchenzl@fudan.edu.cn

${ }^{\dagger}$ Feng-Jiao Wang and Yan-Hua Jing have contributed equally to this article ${ }^{1}$ Department of Integrative Oncology, Fudan University Shanghai Cancer Center, 270 Dong An Road, Shanghai 200032, China

Full list of author information is available at the end of the article
}

excessive deleterious mutations, and an overall frequency approaching TP53 mutations [2]. Recent studies investigated the crucial implication of the SWI/SNF protein complex in the initiation and dedifferentiation of various of neoplasms, which might attribute to its regulation of differentiation and cell proliferation through their enrichment promoters and enhancers active genes [3].

Several SWI/SNF2 family members are known by a SMARCA (SWI/SNF-related Matrix-associated, Actindependent Regulator Chromatin Group A) class or chromatin subgroup remodeler. SMARCA class of chromatin remodeling genes (SMARCAs) play a crucial role in chromatin remodeling, especially in double-strand damage 
repair [4]. SMARCAs are further made up of SMARCA1, SMARCA2, SMARCA3, SMARCA4, SMARCA5, HELLS, SMARCAD1, and SMARCAL1 [5]. Previous studies purposed the SMARCA family members' carcinogenic role in several undifferentiated and differentiated cancers, including lung cancer and renal cell cancer [4]. However, the expression patterns and the exact roles of SMARCAs in pancreatic cancer remained unclear. In this study, the protein and mRNA expression, prognostic values, and potential functions of different SMARCA family members in pancreatic cancer were systematically explored.

\section{Materials and methods \\ ONCOMINE database analysis}

ONCOMINE database (www.oncomine.org) is a publicly accessible online cancer microarray database to collect, standardize, analyze, and deliver cancer transcriptome data to the biomedical research community [6]. The expression levels of SMARCA gene family members in different types of cancer tissues and their adjacent normal samples were identified using ONCOMINE database. The differences were compared by students' $t$-test. The threshold was restricted as follows: $P$ value: 0.01 , fold change: 2 , gene rank: $10 \%$, data type: mRNA.

\section{GEPIA dataset analysis}

Gene Expression Profiling Interactive Analysis (GEPIA) (http://gepia2.cancer-pku.cn/) is a developed interactive web server for estimating the RNA sequencing expression data, based on 9,736 tumors and 8,587 normal samples from the Cancer Genome Atlas (TCGA) and Genotype-Tissue Expression (GTEx) dataset projects. The UCSC Xena project (http://xena.ucsc.edu/) has recomputed all raw RNA-Seq data based on a standard processing pipeline, thus minimizing differences from distinct sources and making such data more compatible. In addition, GEPIA provides essential interactive and customizable functions, including differential expression analysis, profiling plotting, patient survival analysis, similar gene detection, correlation analysis, and dimensionality reduction analysis [7].

\section{Kaplan-Meier plotter}

The prognostic value of mRNA expression of SMARCAs in pancreatic cancers was evaluated using an open online database, Kaplan-Meier Plotter (http://kmplot.com/ analysis/2. This database provided gene expression data and survival information of patients with liver cancer and many other cancer types, such as breast cancer, ovarian cancer, lung cancer, and gastric cancer [8-10]. Patients were divided into high and low expression groups according to the median values of mRNA expression and validated by Kaplan-Meier survival curves with a hazard ratio (HR) with $95 \%$ confidence intervals (CIs). A logrank $P$ value $<0.05$ was considered statically significant.

\section{Immunohistochemistry staining}

The Human Protein Atlas (HPA) (https://www.proteinatl as.org/) is a website that aims to map human proteins in cells, tissues, and organs by utilizing various omics technologies that contain immunohistochemistry-based protein expression, mass spectrometry-based proteomics, transcriptomics, and systems biology [11]. In this study, different SMARCA members' expression between pancreatic cancer and normal tissues was verified by immunohistochemistry image.

\section{Functional enrichment analysis}

Metascape (http://metascape.org) is a free gene-list analysis tool for gene annotation and analysis and an automated meta-analysis tool to understand common and unique pathways within a group of orthogonal target-discovery studies [12]. In this study, the neighboring genes based on TCGA and GTEx expression datasets with similar expression pattern with SMARCA family members were detected using GEPIA, respectively. The selected correlate genes were further used to conduct pathway and process enrichment analysis by Metascape. For this, the Gene Ontology (GO) enrichment analysis, including biological processes (BP), cellular components (CC), and molecular functions (MF) were used for predicting the functional roles of SMARCAs mutations and similar genes associated with SMARCAs mutations. Kyoto Encyclopedia of Genes and Genomes pathways (KEGG) analyzed the SMARCA family members-related pathways and closely related neighbor genes of SMARCAs mutations. A $P$ value cut-off $<0.01$, a minimum overlap of 3 , and a minimum enrichment factor of 1.5 were considered as statistically significant.

\section{Western blotting}

Cells were collected and lysed in cold RIPA buffer supplemented with phenylmethanesulfonyl fluoride (PMSF; Beyotime, China) and centrifuged at $14,000 \mathrm{rpm}$ and $4{ }^{\circ} \mathrm{C}$ for $15 \mathrm{~min}$. Total protein concentrations were determined with a BCA protein assay kit (Beyotime, China). Equal amounts of denatured protein samples were separated by electrophoresis using 10\% SDS-PAGE gel fast preparation kits (Epizyme, China), and then transferred onto $0.45 \mu \mathrm{m}$ polyvinylidene difluoride (PVDF; Millipore, USA) membranes. Subsequently, the membranes were blocked with 5\% BSA diluted in Tris-buffered saline with $0.1 \%$ Tween-20 (TBST) at room temperature. Then, the PVDF membranes were incubated with primary antibodies against $\beta$-actin (1:1000; Proteintech) and HELLS 
(1:1000; Proteintech) at $4{ }^{\circ} \mathrm{C}$ overnight. The next day, the primary antibody was washed three times with TBST and incubated with HRP-conjugated secondary antibodies at room temperature for $1 \mathrm{~h}$.

\section{Cell culture and HELLS knockdown}

The human pancreatic cancer cell lines were obtained from the American Type Culture Collection (ATCC, USA), and cultured in Dulbecco's modified Eagle's medium (DMEM) supplemented with 10\% fetal bovine serum (FBS), $100 \mu \mathrm{g} / \mathrm{ml}$ penicillin and $100 \mu \mathrm{g} / \mathrm{ml}$ streptomycin in a humidified incubator at $37^{\circ} \mathrm{C}$ under an atmosphere with $5 \% \mathrm{CO} 2$.

The packaged lentivirus containing shRNAs targeting HELLS (shHELLS-1, 5'-AACAAGGCGATAAACAAC AAC-3'; shHELLS-2, 5'-TTCTACAGGGATATTCAC TTC-3'; shHELLS-3, 5'-AATTGTTTCTTTCTCACT GGA-3') and a negative control sequence (shHELLS-con, $5^{\prime}$-TTCTCCGAACGTGTCACGT-3') were designed by GENE (Shanghai, China). Cells were seeded in 24-well plates and cultured for $24 \mathrm{~h}$. Then, shRNAs were transfected using transfection reagent provided by GENE. After incubation of $72 \mathrm{~h}$ to $80 \%$ confluence, cells were selected in puromycin and the knockdown efficiency of HELLS was confirmed by quantitative real-time PCR (qRT- PCR) and western blotting.

\section{RNA extraction and qRT- PCR}

Total RNA was extracted from cells using RNAiso Plus reagent (Takara, Tokyo, Japan). The cDNA was synthesized using Takara PrimeScript ${ }^{\mathrm{TM}}$ RT Master Mix. qRTPCR was performed with TB Green ${ }^{\circledR}$ Premix Ex Taq ${ }^{\text {TM }}$ using an ABI 7900HT Real-Time PCR system (Applied Biosystems, CA, USA). The relative gene mRNA expressions were calculated by $2^{-\Delta \Delta C t}$ method with respect to GAPDH. The primer sequences are listed in Table 1.

\section{Cell proliferation assay}

A cell counting kit-8 (CCK-8; DOJINDO, China) was used to measure cell proliferation. In brief, cell suspensions at a density of $3 \times 10^{3}$ cells per $200 \mu$ l were seeded in 96-well plates and incubated for $0,24,48,72,96 \mathrm{~h}$. After the indicated incubation times, the medium was removed and a CCK- 8 DMEM solution (10 $\mu \mathrm{l}$ of CCK-8

Table 1 The sequences of primers

\begin{tabular}{ll}
\hline Primers & Sequences $\left(\mathbf{5}^{\prime} \mathbf{-} \mathbf{3}^{\prime}\right)$ \\
\hline HELLS Forward & ACTCCTCCTCTACTAATCTCTG \\
HELLS Reverse & GGCTGACCATTACACTTCC \\
GAPDH Forward & GCACCGTCAAGGCTGAGAAC \\
GAPDH Reverse & TGGTGAAGACGCCAGTGGA \\
\hline
\end{tabular}

in $100 \mu \mathrm{l}$ of DMEM) was added into each well and incubated at $37{ }^{\circ} \mathrm{C}$ for an additional $2 \mathrm{~h}$. The absorbance of each well at $450 \mathrm{~nm}$ were measured using a microplate reader.

\section{Colony formation assay}

A total of 500 cells per well were seeded in 6-well plates and incubated for 14 days. After that, cell colonies were washed with phosphate-buffered saline (PBS) three times, fixed with $4 \%$ paraformaldehyde for $30 \mathrm{~min}$, and further stained with $0.1 \%$ crystal violet solution for $15 \mathrm{~min}$. Cell visible colonies containing more than 50 cells were captured by mobile camera.

\section{Cell cycle analysis}

The cell cycle was examined by flow cytometric analysis with propidium iodide (PI)/RNase staining kit (Beyotime, China). Cell suspensions at a density of $3 \times 10^{5}$ cells/well were seeded in 6-well plates and incubated overnight. After cycle synchronization by serum starvation for $24 \mathrm{~h}$, cells were cultured in regular DMEM for $48 \mathrm{~h}$. Then, cells were washed in cold PBS and centrifuged at $1000 \times g$ for $5 \mathrm{~min}$, and resuspended in ice-cold $70 \%$ ethanol for at least $2 \mathrm{~h}$ on ice. Centrifuged, decant and washed again in cold PBS. About $1 \times 10^{6}$ cells were resuspended in $0.5 \mathrm{ml}$ working solution per sample $(500 \mu \mathrm{l}$ of assay buffer mixed $25 \mu \mathrm{l}$ of PI solution and $2.5 \mu \mathrm{l}$ RNase solution), and kept for $30 \mathrm{~min}$ at $37^{\circ} \mathrm{C}$ and $4{ }^{\circ} \mathrm{C}$ in the dark, respectively.

\section{Statistical analysis}

Statistical analysis was performed by GraphPad Prism (version 7.0a), and the results are presented as the means \pm SD. Two-tailed unpaired Student's $t$-test, and one-way or two-way ANOVA were used to evaluate the data. $P$-value less than 0.05 was considered statistically significant.

\section{Results}

Transcriptional levels and protein expression of SMARCAs in pancreatic cancer patients

Eight SMARCA family members have been identified in mammalian cancers. The different transcriptional levels of SMARCAs in pancreatic cancer and normal samples were analyzed by ONCOMINE database (Fig. 1; Table 2). Results revealed that the mRNA levels of SMARCA3, SMARCA4, HELLS were significantly upregulated in patients with pancreatic cancer, while the mRNA levels of SMARCA2 and SMARCAD1 were downregulated (Fig. 1). In Table 2, the results showed that the transcription levels of SMARCA3 were significantly higher in patients with pancreatic cancer in Buchholz's dataset [13]. SMARCA3 was overexpressed in pancreatic ductal adenocarcinoma compared to the normal tissues, with 


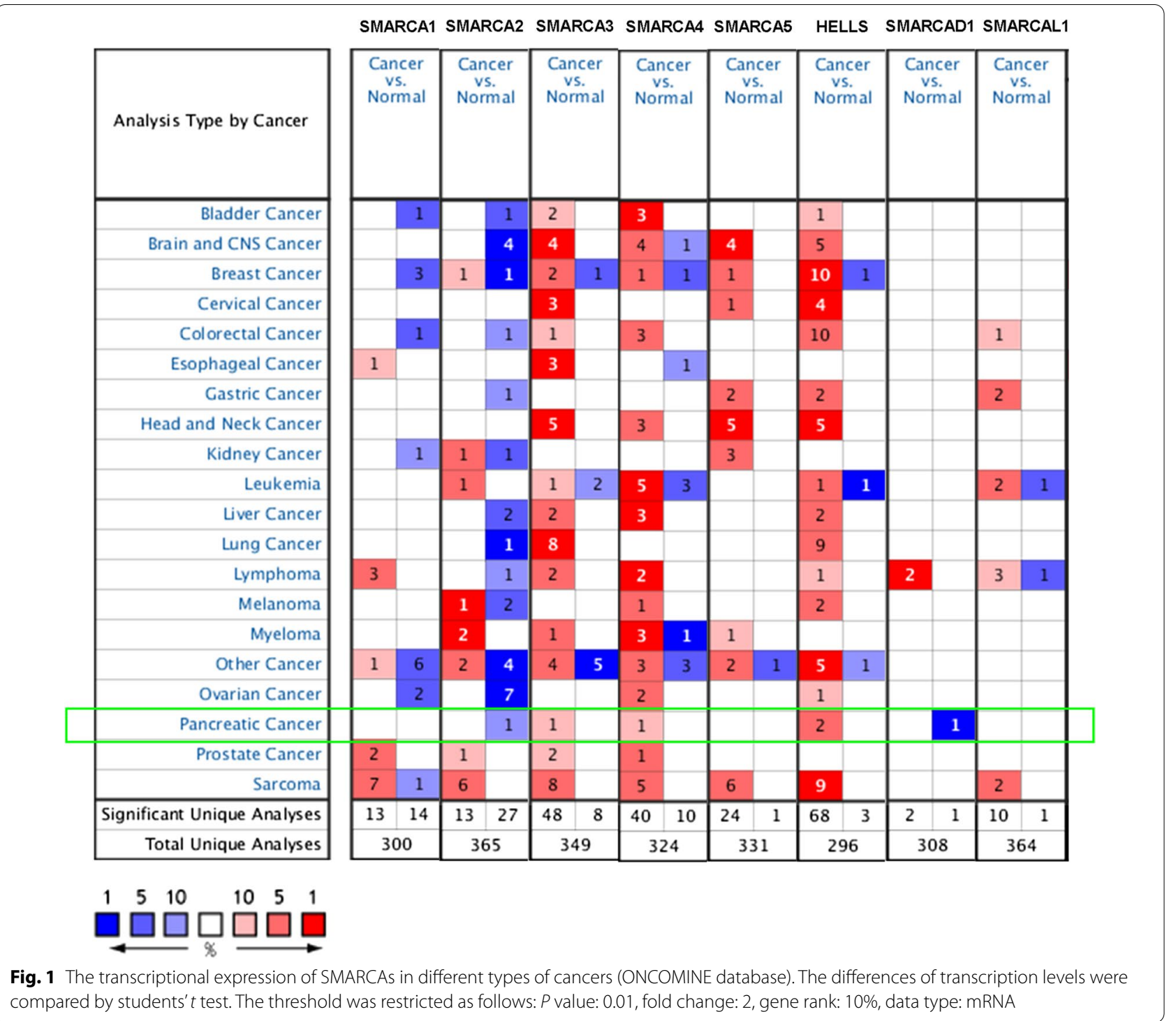

Table 2 SMARCAs transcription levels between pancreatic cancer and normal tissues (ONCOMINE)

\begin{tabular}{llllll}
\hline & $\begin{array}{l}\text { Types of pancreatic cancer versus } \\
\text { normal pancreatic tissue }\end{array}$ & Fold change & P value & t test & References \\
\hline SMARCA2 & Pancreatic ductal adenocarcinoma & -6.555 & 0.009 & 3.107 & Buchholz Pancreas [13] \\
SMARCA3 & Pancreatic ductal adenocarcinoma & 2.522 & 0.008 & 3.688 & Buchholz Pancreas [13] \\
SMARCA4 & Pancreatic adenocarcinoma & 2.257 & $7.16 \mathrm{E}-4$ & 4.459 & Logsdon Pancreas [14] \\
HELLS & Pancreatic ductal adenocarcinoma & 2.596 & 0.005 & 2.867 & Grutzmann Pancreas [15] \\
& Pancreatic carcinoma & 2.386 & $3.50 \mathrm{E}-8$ & 6.352 & Pei Pancreas [16] \\
\hline
\end{tabular}

Order by Over-expression: Fold Change; $P$ value $<0.01$; Fold Change $>2$; Gene Rank: Top 10\%

a 2.522 fold-change. In Logsdon's dataset [14], the transcription levels of SMARCA4 were significantly higher in patients with pancreatic adenocarcinoma, with a fold change of 2.257 and a $P$ value of $7.16 \mathrm{E}-4$. HELLS was highly expressed in two datasets $[15,16]$. HELLS was overexpressed in pancreatic ductal adenocarcinoma compared with that in the normal samples in Grutzmann's dataset [15], with a fold change of 2.596 and a $P$ 
A.

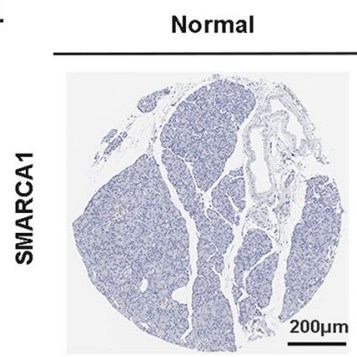

Staining: Not detected Antibody: HPA003335

c.

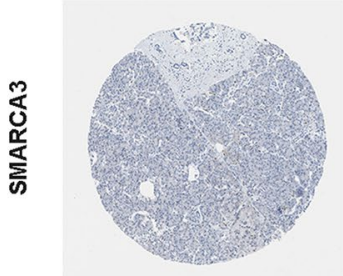

Staining: Not detected

Antibody: HPA015284

E.

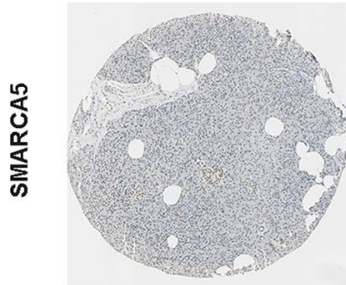

Staining: Medium Antibody: HPA008751

G.

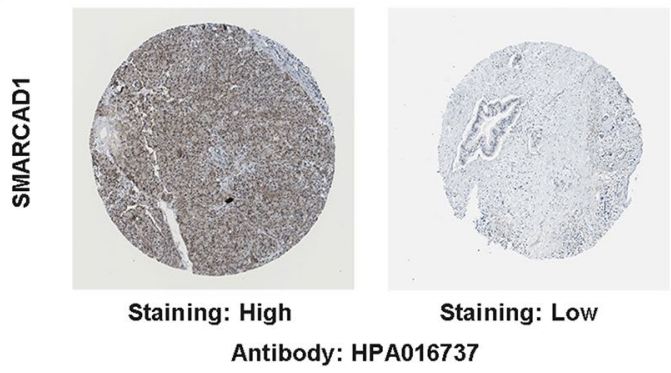

B.

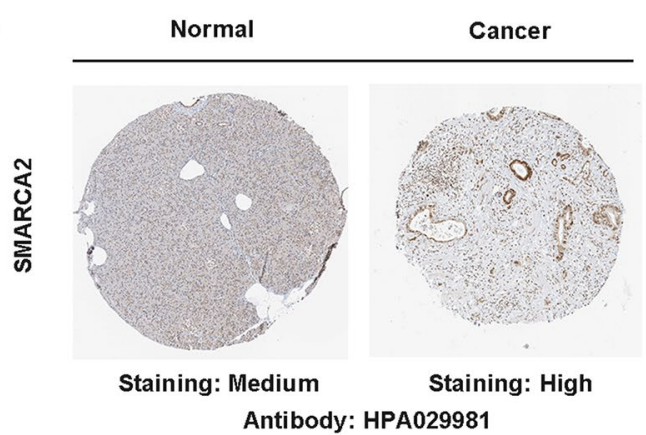

D.

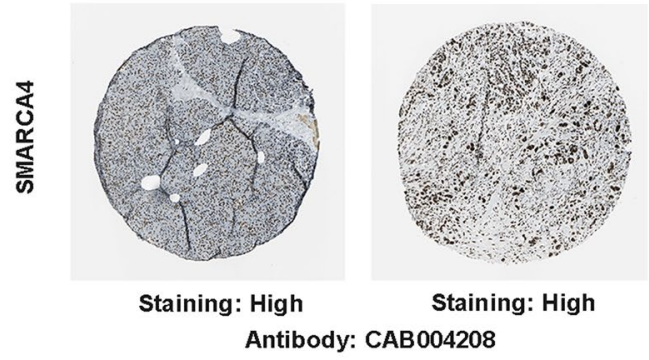

F.

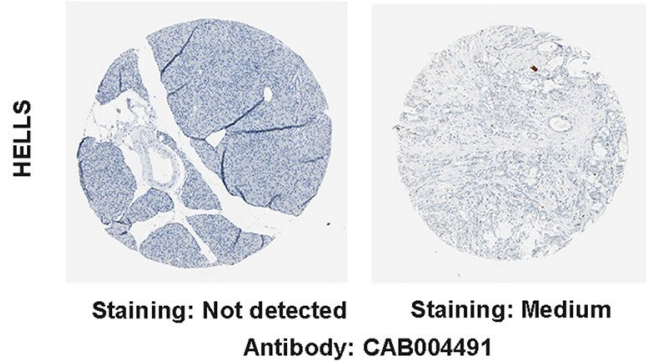

H.

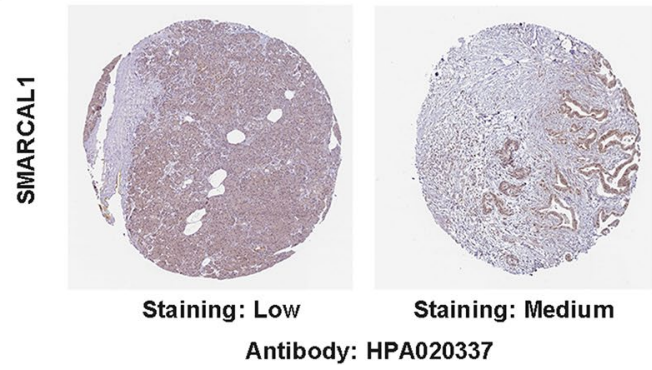

Fig. 2 Protein expression of SMARCAs obtained from the Human Protein Atlas (HPA) online database (scale bar, 200 um). Staining was divided into not detected, low, medium and high based on the intensity of staining. A SMARCA1 protein was not detected in normal tissues and pancreatic cancer tissues; B, E, H SMARCA2/5 and SMARCAL1 protein were expressed medium or low in normal tissues, while high or medium expression were observed in pancreatic tissues; $\mathbf{C}, \mathbf{F}$ SMARCA3 and HELLS protein were not observed in normal pancreatic tissues, whereas low and medium expression were detected in pancreatic cancer tissues, respectively; D SMARCA4 protein was expressed high both in normal tissues and pancreatic cancer tissues; G SMARCAD1 protein was lower in pancreatic cancer tissues compared to normal tissues

value of 0.005. In Pei's dataset [16], HELLS was overexpressed in pancreatic cancer with a fold change of 2.386 and a $P$ value of $3.50 \mathrm{E}-8$. However, SMARCA2 was found significantly decreased in patients with pancreatic cancer compared with that in the normal samples in Buchholz's dataset [13], with a fold change of -6.555 and a $P$ value of 0.009 .
Additionally, after examining the transcriptional levels of SMARCAs in pancreatic cancer, we explored the protein expression of SMARCAs using the HPA online database. We found that SMARCA3 and HELLS proteins were not observed in normal pancreatic tissues, whereas low and medium expressions were detected in pancreatic cancer tissues, respectively (Fig. 2C, F). However, 
lower protein expressions of SMARCAD1 was observed in pancreatic cancer tissues compared to normal tissues (Fig. 2G). SMARCA2/5 and SMARCAL1 protein were expressed medium or low in normal tissues, while high or medium expression was observed in pancreatic tissues (Fig. 2B, E, H). High protein expression of SMARCA4 was observed both at normal pancreatic tissues and cancer tissues (Fig. 2D), while SMARCA1 was not detected in both of them (Fig. 2A).

\section{Association between the mRNA expression of SMARCAs and the clinicopathological parameters of patients with pancreatic cancer}

We compared the mRNA Levels of SMARCA family members between pancreatic cancer tissues and normal tissues using the GEPIA. In our study, the results demonstrated the higher transcripts per million (TPM) levels of SMARCA1, SMARCA2, SMARCA4, SMARCA5, HELLS, and SMARCAL1 in pancreatic cancer samples than that in normal control $(P<0.05)$, which suggests that the above six genes possessed more transcripts in pancreatic cancer tissues (Fig. 3A). Consistent with the mRNA levels of SMARCA1, SMARCA2, SMARCA4, SMARCA5, HELLS, and SMARCAL1, a significant difference was found in patients with pancreatic cancer $(P<0.05)$, and the mRNA levels of SMARCA3 and SMARCAD1 displayed no significant difference between the pancreatic cancer group and the control group $(P>0.05)$ (Fig. 3B). Subsequently, we analyzed the correlation between the mRNA expression of SMARCAs and tumor stages in pancreatic cancer. The mRNA expression levels of SMARCA1, SMARCA2, and SMARCA3 were remarkably correlated with the tumor stages $(P<0.05)$ (Fig. 4A-C), whereas the mRNA expression of SMARCA4, SMARCA5, HELLS, SMARCAD1, and SMARCAL1 did not differ significantly $(P>0.05)$ (Fig. 4D-H).

\section{Prognostic value of mRNA expression of SMARCAs in pancreatic cancer}

Further, we explored the potential value of SMARCAs in the survival of patients with pancreatic cancer using Kaplan-Meier Plotter tools. The Kaplan-Meier curve and log-rank test analyses showed that mRNA expression of SMARCA1, SMARCA2, SMARCA3, HELLS, and SMARCAD1 were significantly correlated with patients' OS (Fig. 5A-C, F, G). To be more specific, the high mRNA levels of SMARCA1 (HR $=0.5,95 \%$ CI 0.32-0.77, and $P=0.0015)$, SMARCA2 (HR $=0.56$, 95\% CI $0.37-$ 0.85 , and $P=0.0057)$, SMARCA3 ( $\mathrm{HR}=0.63,95 \% \mathrm{CI}$ $0.41-0.98$, and $P=0.039)$ and SMARCAD1 $(\mathrm{HR}=0.63$, 95\% CI $0.42-0.95$, and $P=0.026)$ may contribute to favorable prognosis of pancreatic cancer $(P<0.05)$, while the mRNA expression of HELLS was negatively correlated with patients' OS $(\mathrm{HR}=1.77,95 \%$ CI $1.09-2.86$, and $P=0.019)$. However, the mRNA expression of SMARCA4, SMARCA5, and SMARCAL1 showed no significant correlation with the prognosis of pancreatic cancer $(P>0.05)$ (Fig. 5D, E, H). The above results indicated that high mRNA expressions of SMARCA1/2/3 and SMARCAD1, or low mRNA levels of HELLS were significantly associated with longer OS, which may be exploited as potential prognostic biomarkers for pancreatic cancer.

\section{Predicted functions and pathways of alterations in SMARCAs and their similar genes in pancreatic cancer} A total of 240 similar genes based on TCGA and GTEx expression datasets were detected by GEPIA, which were significantly correlated with SMARCAs alterations. The functions and pathways of alterations in SMARCAs and their similar genes were conducted by analyzing GO and KEGG in Metascape. The results showed that BP, such as GO: 0051301 (cell division), GO: 0071103 (DNA conformation change), GO: 0000281 (mitotic cytokinesis), and GO: 0044770 (cell cycle phase transition) were significantly regulated by the SMARCAs alterations in pancreatic cancer (Fig. 6A). CC, including GO: 0005815 (microtubule organizing center), GO: 0030496 (midbody), GO: 0000775 (chromosome, centromeric region), GO: 0016604 (nuclear body) and GO: 0005819 (spindle) were significantly associated with the SMARCAs mutations (Fig. 6B). In addition, SMARCAs alterations prominently affected the MF, including GO: 0003682 (chromatin binding) and GO: 0008017 (microtubule binding) (Fig. 6C). Among the KEGG pathways, hsa04110 (Cell cycle) and hsa04330 (Notch signaling pathway) were prominently involved in the tumorigenesis and pathogenesis of pancreatic cancer (Fig. 6D).

\section{Downregulation of HELLS suppresses pancreatic cancer cells proliferation, colony formation, and affects cell cycle distribution}

Notably, among the SMARCA family members, data mining analysis showed that mRNA and protein levels of HELLS are negatively correlated with outcomes in pancreatic cancer patients, which is highly in accordance with its oncogenic roles in previous literature. According to the abovementioned bioinformatic analysis results, HELLS could serve as a potential prognostic biomarker of PDAC. Therefore, cell function experiments were performed to verify its potential effect on pancreatic cancer. We firstly investigated the expression of HELLS in pancreatic cancer cell lines and normal human pancreatic ductal epithelial cells (HPDE). The results showed that the protein levels of HELLS were highly upregulated in PANC-1 cells than in HPDE cells (Fig. 7A). To determine 
A.
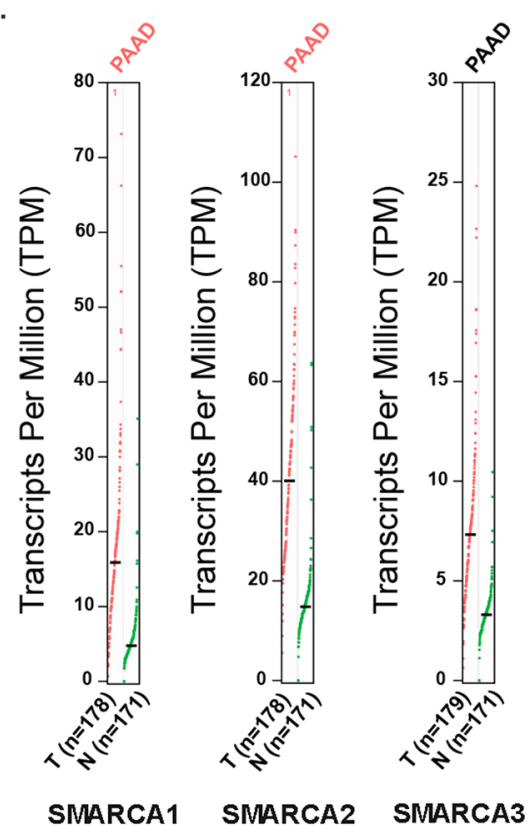

SMARCA3

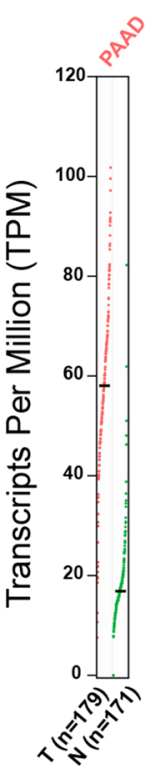

SMARCA4
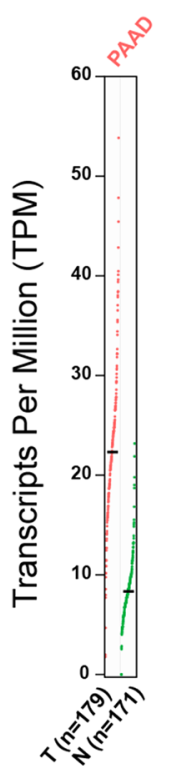

SMARCA5
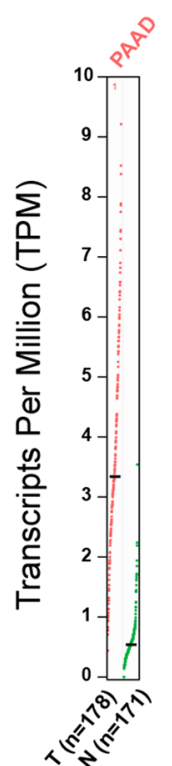

HELLS

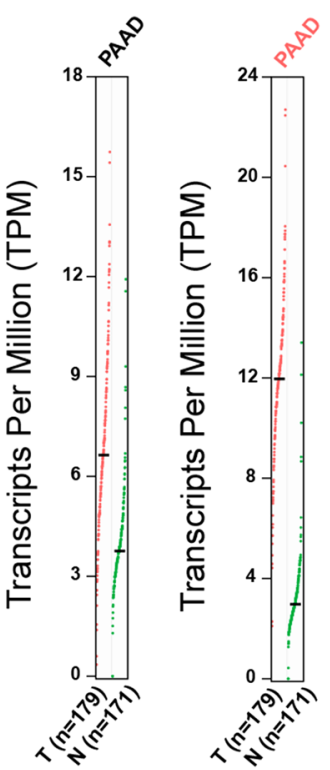

SMARCAD1 SMARCAL1

B.

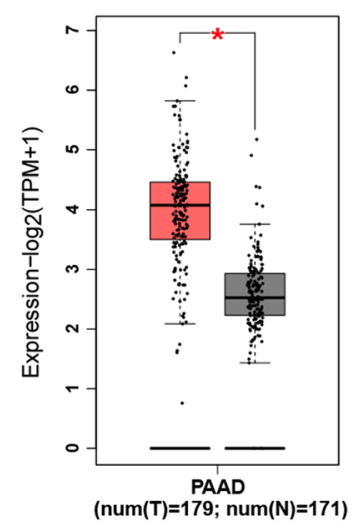

SMARCA1

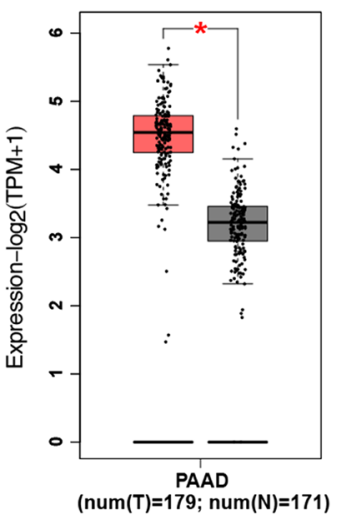

SMARCA5

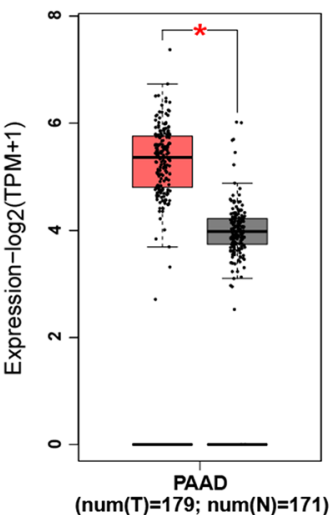

SMARCA2

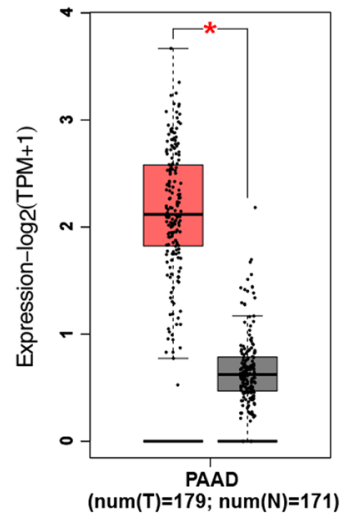

HELLS

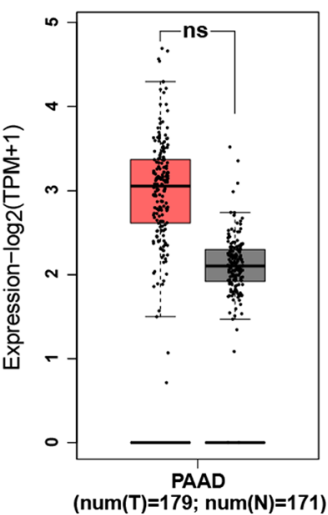

SMARCA3

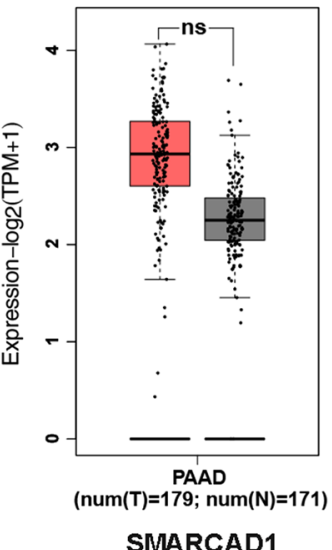

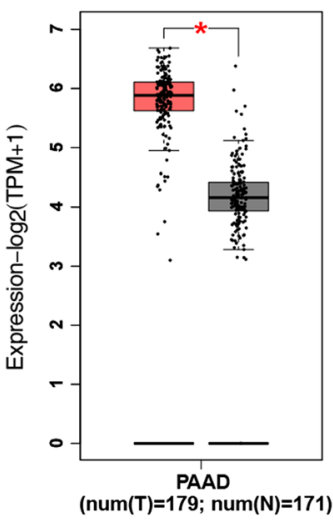

SMARCA4

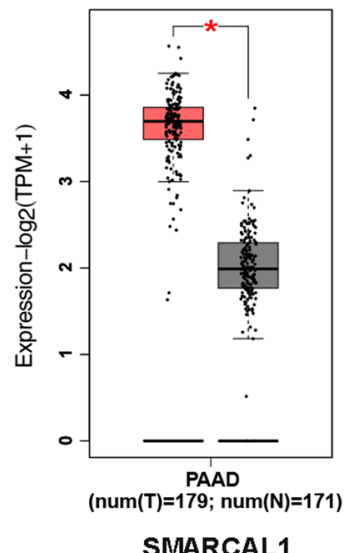

Fig. 3 The expression of SMARCAs between pancreatic cancer tissues and normal tissues (GEPIA). A The transcripts per million (TPM) levels of SMARCAs between pancreatic cancer tissues and normal tissues; $\mathbf{B}$ The mRNA levels of SMARCAs between pancreatic cancer tissues and normal tissues 

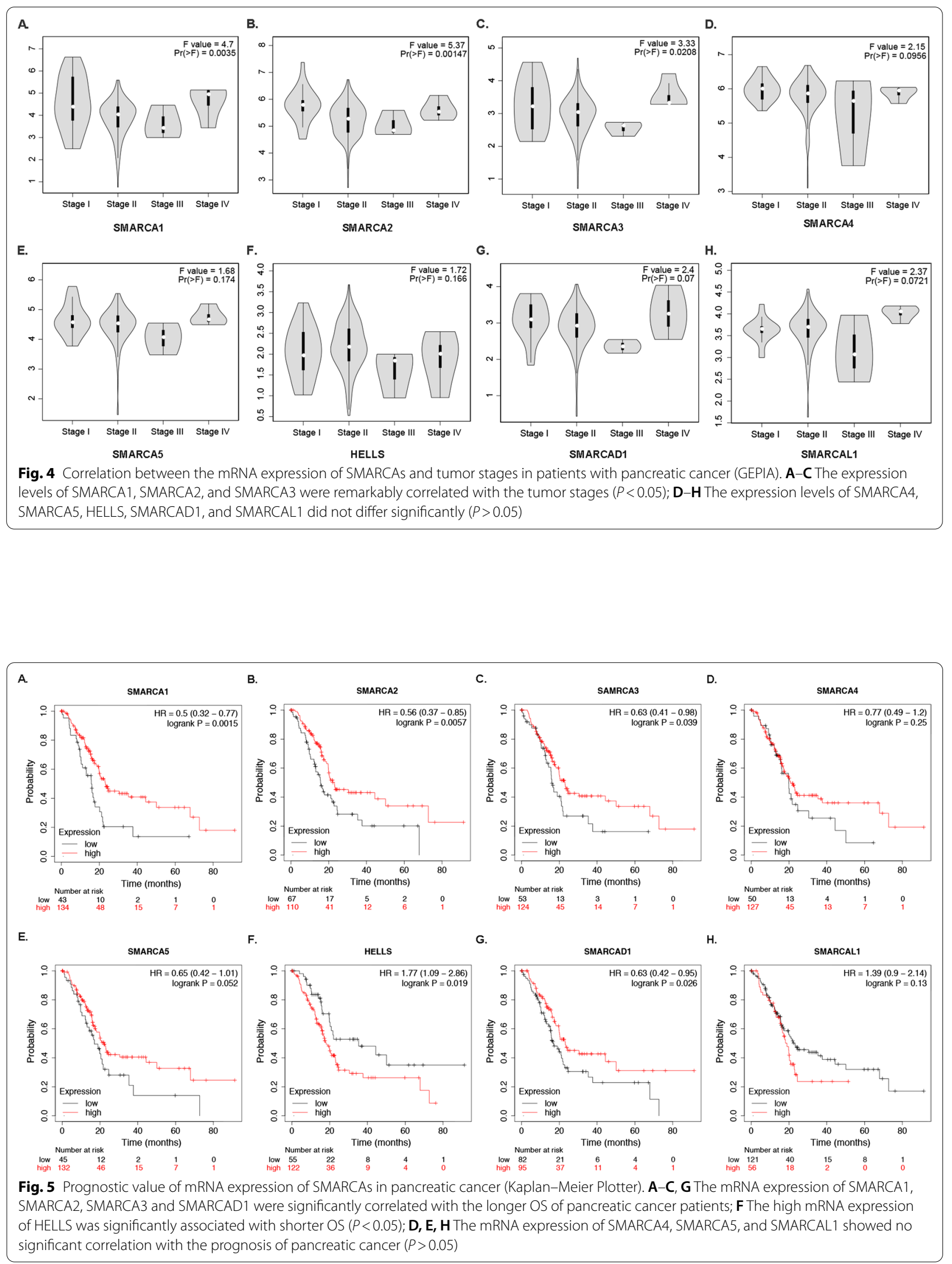


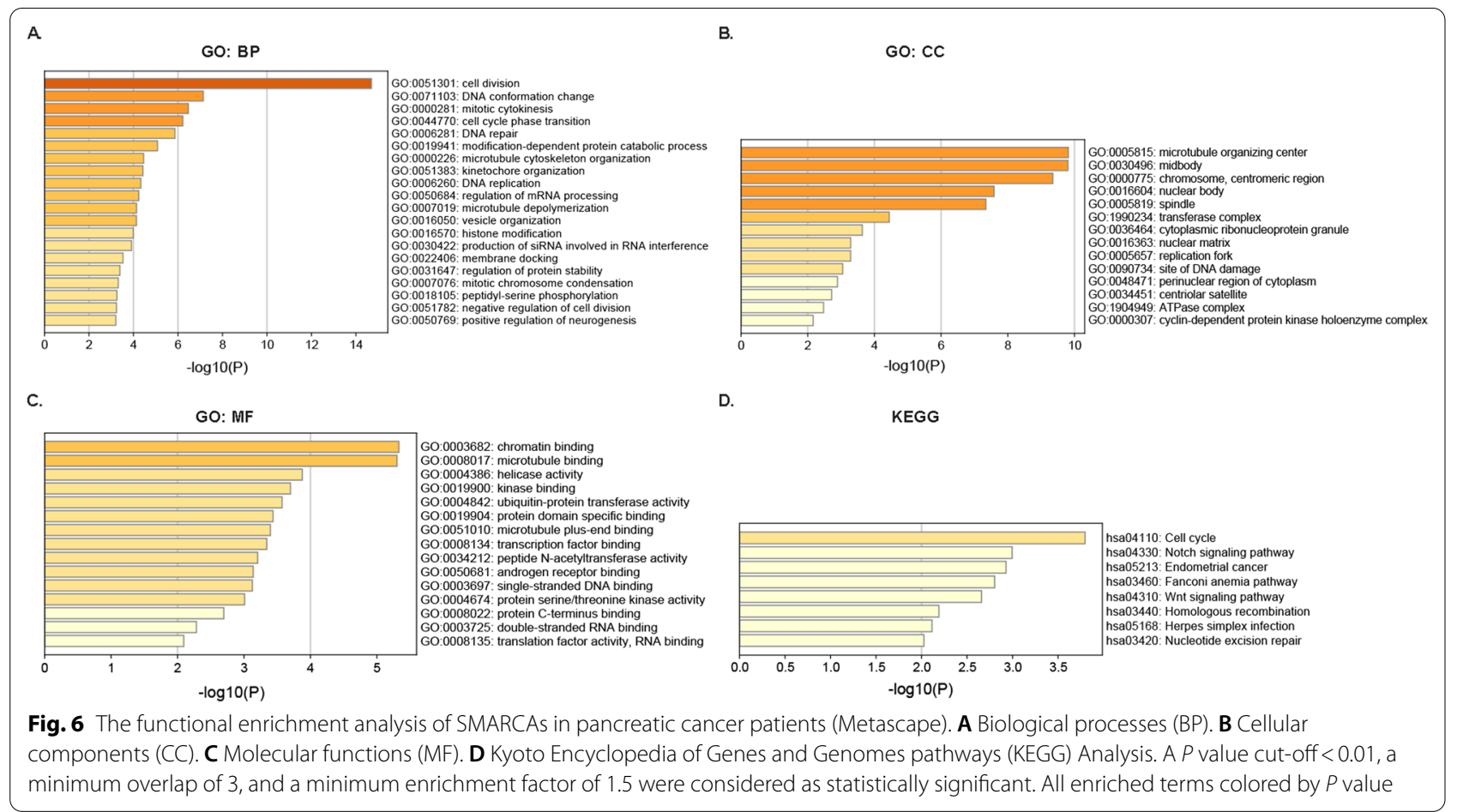

the oncogenic roles of HELLS in pancreatic cancer, we stably knocked down HELLS in PANC-1 cells, and verified at mRNA and protein levels (Fig. 7B). The results showed that the downregulation of HELLS significantly inhibited cell proliferation $(P<0.05$, Fig. $7 C)$, and dramatically impaired the ability of the colony formation (Fig. 7D). Thus, we further investigated cell cycle by flow cytometry to examine whether HELLS expression affected cycle distribution in pancreatic cancer (Fig. 7E). As shown in Fig. 7F, $54.16 \pm 1.568 \%$ of shHELLS-1, $47.73 \pm 1.898 \%$ of shHELLS-2, $55.81 \pm 2.127 \%$ of shHELLS-3, and $34.52 \pm 1.817 \%$ of shHELLS-con were in $\mathrm{S}$ phase. The statistical data showed a significant increase in $\mathrm{S}$ fraction in shHELLS group compared with the shHELLS-con group $\left.{ }^{* * * * * *} P=0.0001\right)$, indicating that knockdown of HELLS inhibited cell proliferation by arresting cells at $S$ phase in pancreatic cancer.

\section{Discussion}

SMARCA1 is a tumor-suppressor gene located on chromosome X [17]. Until now, the function of SMARCA1 in the type of cancers has not been a focus of research. Little was known about the physiological functions of SMARCA1 in pancreatic cancer. In our study, conflicting findings of the roles of SMARCA1 in pancreatic cancer were observed. On the one hand, high SMARCA1 mRNA expression was observed in pancreatic cancer, and SMARCA1 mRNA expression was remarkably correlated with tumor stages. On the other hand, high mRNA SMARCA1 expression was significantly correlated with favorable OS in patients with pancreatic cancer. Therefore, further studies consist of larger sample sizes worth to validate SMARCA1 expression patterns in patients with pancreatic cancer and to explore the roles of SMARCA1 in tumorigenesis.

SMARCA2 (also known as BRM) is one of two evolutionarily conserved catalytic ATPase subunits of SWI/ SNF complexes sharing a high degree of amino acid sequence identity with SMARCA4 and interacting with transcription factors and other cellular proteins to modulate transcription activity of multiple genes [18]. Wilson BG et al. has demonstrated a compensatory role between SMARCA2 and SMARCA4, that a reciprocal assembly of SMARCA2 into SWI/SNF complexes when SMARCA4 is genetic inactive, providing insight into the mechanisms driving tumorigenesis [19]. SMARCA2 plays important roles in cell proliferation, linage specification and development, cell adhesion, cytokine responses, and DNA repair, which was previously implicated in risk and prognosis in lung, esophageal, colon cancer, and pancreatic cancer $[18,20]$. However, conflicting evidence about its roles in various type of cancers has remained. On one hand, frequent loss of SMARCA2 expression was observed in patients with lung cancer and gastric cancer. It correlates with cancer aggressiveness and poor prognosis, suggesting a suppressive role of SMARCA2 
A.

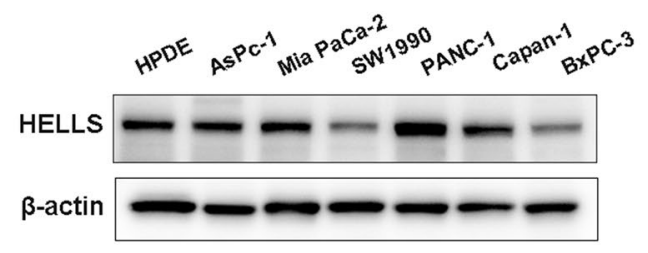

B.

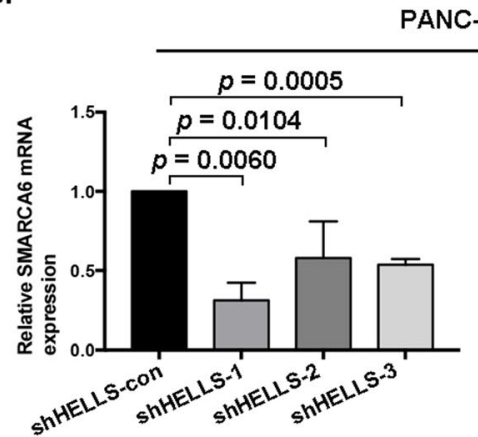

c.

PANC-1

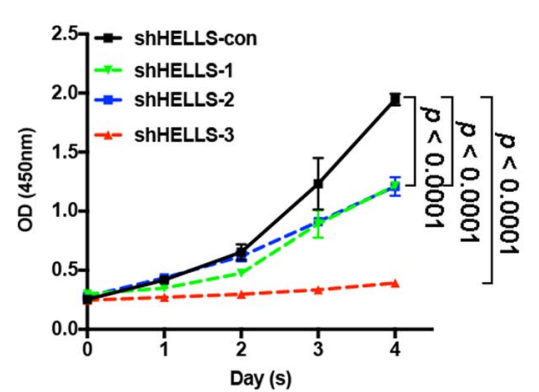

E.

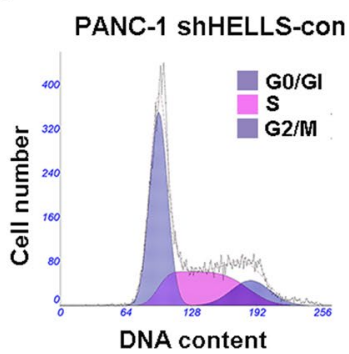

PANC-1 ShHELLS-2

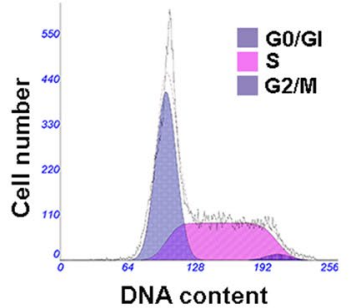

PANC-1 ShHELLS-1

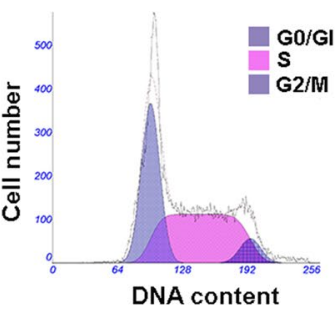

PANC-1 ShHELLS-3

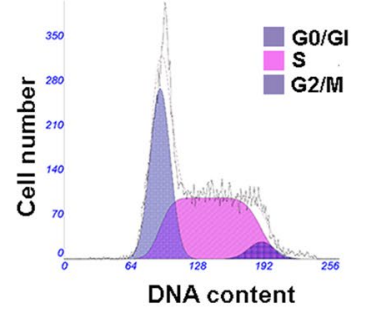

F.

PANC-1

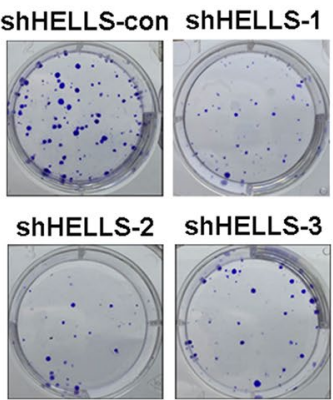

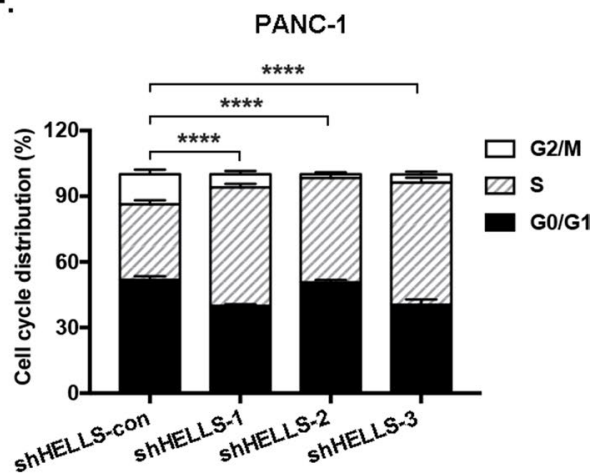

Fig. 7 Downregulation of HELLS suppresses pancreatic cancer cells proliferation, colony formation, and affects cell cycle distribution. A The expression of HELLS in pancreatic cancer cell lines and normal human pancreatic ductal epithelial cells (HPDE). The grouping of blots cropped from different parts of the same gel, or from different gels, fields, or exposures was divided with white space. B Verification at mRNA and protein levels of shHELLS in PANC-1 cells. The grouping of blots cropped from different parts of the same gel, or from different gels, fields, or exposures was divided with white space. C, D Downregulation of HELLS significantly inhibited cell proliferation, and impaired the ability of the colony formation $(P<0.05)$. E Cell cycle distribution under different shHELLS analyzed by flow cytometry. $\mathbf{F}$ A significant increase in $S$ fraction in shHELLS group compared with the shHELLS-con group $\left.{ }^{* * * *} P=0.0001\right)$

in these tumors [21, 22]. On the other hand, SMARCA2 overexpression was found in human epithelial ovarian cancer. Xu X et al. reported a relatively higher expression of SMARCA2 in cisplatin-resistant ovarian cancer cells, leading to a significant reduction in apoptosis indicative of resistance to cisplatin [23]. Similar to SMARCA1, conflicting observations about SMARCA2 were also found in our study. Therefore, further studies are worth to evaluate the precise roles of SMARCA2 in pancreatic cancer.

SMARCA3 (also known as Helicase-like transcription factor, HLTF) is frequently observed in colorectal cancer $(\mathrm{CRC})$ and is negatively associated with the progression of CRC [24]. Consistent with the conclusion, in our study, despite no statistically significant difference in mRNA between pancreatic cancer and normal tissues, mRNA expression of SMARCA3 was significantly correlated with tumor stages, and high SMARCA3 mRNA expression was positively related to longer OS in pancreatic cancer. Of note, protein expression of SMARCA3 were observed in pancreatic cancer tissues, but not in normal tissues. Given that, further verifications are required to determine the mRNA and protein expression of SMARCA3 between pancreatic cancer and normal tissues. 
Studies have pointed to a role for human SMARCAD1 in genomic instability during the past few years, which can lead to cell death or cancer development in higher eukaryotes [25]. On the one hand, knockdown of SMARCAD1 resulted in a significant decrease in breast cancer cell proliferation and colony formation, mainly through a potent inhibition of STAT3 phosphorylation [26]. The conclusion was consistent with the study from $\mathrm{Al}$ Kubaisy et al. [27], which also suggested a possible tumor oncogenic role for SMARCAD1. However, the expression of SMARCAD1 in patients with bladder cancer was associated with an increased survival time [28]. Anti-oncogenic properties of SMARCAD1 was also shown in HCC [25]. These data suggested that SMARCAD1 as a tumor suppressor. Notably, the role of SMARCAD1 seems conflicting in pancreatic cancer. The previous study has indicated that SMARCAD1 is highly expressed in pancreatic cancer tissues and negatively correlated with survival time. Mechanistically, SMARCAD1 promotes pancreatic cancer cell growth and metastasis via activating Wnt/ $\beta$ catenin-mediated EMT [29]. On the contrary, in our study, we found that SMARCAD1 expressed lower in pancreatic cancer tissues than that in normal tissues, and significantly associated with unfavorable OS.

In addition to SMARCA2, SMARCA4 is another exclusive catalytic subunit of SWI/SNF complexes containing a bromodomain and an ATPase domain essential for the modulation of SWI/SNF chromatin remodeling $[3,30]$. A recent study indicated that SMARCA4 functioned as a bona fide tumor suppressor and cooperatd with p53 loss and Kras activation [31]. SMARCA4 is frequently mutated in multiple cancer types, including non-small cell lung carcinoma (NSCLC) (10-35\%), Burkitt's lymphoma (15\%) and childhood medulloblastoma (5-10\%), and occasionally mutated in pancreatic adenocarcinoma, ovarian clear cell carcinoma and melanoma $[19,32]$. Jelinic $P$ et al. revealed that inactivating mutations in SMARCA4 were associated with poor lung adenocarcinoma outcomes. Moreover, re-expression of SMARCA4 through electroporation in SMARCA4null H1299 NSCLCs resulted in a dose-dependent suppression of cell growth [33]. As for SMARCA5, it was previously observed overexpressed in many malignant neoplasms. Jin et al. found overexpression of mRNA and protein in breast cancer, significantly associated with TNM stages, tumor size, high proliferation index, and poor OS [34]. Consistent with the above conclusions, Wang $\mathrm{H}$ et al. reported that survival rates among patients with gliomas exhibiting high SMARCA5 expression were much poor than that low levels [35]. These data suggested that SMARCA5 might be a novel prognostic biomarker in those cancers. SMARCAL1 is implicated in cellular DNA replication stress, such as stabilization of
DNA replication forks and inhibition of genome instability and tumorigenesis induced by oncogenes [36]. In this report, Puccetti MV et al. biologically demonstrate that loss of SMARCAL1 profoundly suppressed Myc-driven $B$ cell lymphomagenesis, suggesting that SMARCAL1 is could provide a therapeutic opportunity in Myc-driven malignancies. In our study, although high mRNA expressions of SMARCA4/5/SMARCAL1 were all observed in pancreatic cancer patients compared to normal tissues, no significant correlation was found between those three SMARCA members and tumor stages and OS.

Significant overexpression of HELLS has been found in various cancers, including medulloblastoma, hepatocellular carcinoma (HCC), and CRC. Literature from Law $\mathrm{CT}$ et al. reported that HELLS was remarkably overexpressed in HCC and positively correlated with aggressive clinicopathological features and poorer prognosis than patients with lower HELLS expression, which was further confirmed by reduced HCC growth and metastasis both in vitro and in vivo following the depletion of HELLS. Moreover, the inactivation of HELLS resulted in metabolic reprogramming and reversed the warburg effect in HCC cells [37]. Parallel to this finding, Zhang G et al. found that HELLS expression was highly expressed in glioblastoma and positively associated with glioma progression. The oncogenic roles of HELLS in glioblastoma are likely mediated through interactions with E2F3 and MYC [38]. The above observation revealed that HELLS is a key epigenetic regulator driving those tumors' pathogenesis. In our study, bioinformatics methods were used to detect potential prognostic biomarkers for challenging pancreatic cancer. Consistently, significantly higher mRNA and protein expressions of HELLS were observed in pancreatic cancer tissues compared to normal tissues. Although HELLS showed no statistically significant association with patients' tumor stages, HELLS overexpression is significantly correlated with the poor OS. In addition, downregulation of HELLS inhibits pancreatic cancer cells proliferation and colony formation, and induces cell cycle arrest in vitro.

In addition to SMARCA family members, a highly conserved core subunit (SNF5, also known as SMARCB1) is present in all known variants of the SWI/ SNF complex [39]. It has been reported that its mutation can evoke powerful genome-wide downstream effects, which may be counteracted therapeutically [40]. INI1, encoded by SMARCB1, functions as a tumor suppressor. Loss of INI1 expression leads to oncogenic activation of EZH2 (an enzyme that catalyzes trimethylation of histone H3 lysine 27, H3K27me3), especially in epithelioid sarcoma [41]. Even though EZH2 has been pursued as a therapeutic target for several types of tumors, such as sarcoma, lymphoma, and 
malignant rhabdoid tumor (MRT) [42], there is still much unknown how SMARCB1/INI1 drives EZH2 or how SMARCB1 interacts with the potential targets in pancreatic cancer. It would be interesting to explore their underlying mechanism and potential roles further, which would undoubtedly provide insights into a valuable link between chromatin remodelelling and pancreatic cancer.

In summary, we systematically analyzed the expression and prognostic value of SMARCAs in pancreatic cancer. Functionally, the data demonstrated that HELLS plays vital roles in promoting pancreatic cancer progression, and serves as a poor prognostic biomarker for pancreatic cancer.

\section{Abbreviations}

SWI/SNF: Switch/sucrose nonfermenting complex; GEPIA: Gene Expression Profiling Interactive Analysis; TCGA: The Cancer Genome Atlas; GTEx: Genotype-Tissue Expression; HR: Hazard ratio; Cls: Confidence intervals; HPA: Human Protein Atlas; GISTC: Genomic Identification of Significant Targets in Cancer; OS: Overall survival; DFS: Disease-free survival; GO: Gene ontology; BP: Biological processes; CC: Cellular components; MF: Molecular functions; KEGG: Kyoto Encyclopedia of Genes and Genomes; PMSF: Phenylmethanesulfonyl fluoride; TBST: Tris-buffered saline with 0.1\% Tween-20; ATCC: American Type Culture Collection; DMEM: Dulbecco's modified Eagle's medium; FBS: Fetal bovine serum; qRT- PCR: Quantitative real-time PCR; CCK-8: Cell counting kit-8; PBS: Phosphate-buffered saline; PI: Propidium iodide; TPM: Transcripts per million; HPDE: Human pancreatic ductal epithelial cells; HLTF: Helicase-like transcription factor; CRC: Colorectal cancer; NSCLC: Non-small cell lung carcinoma; HCC: Hepatocellular carcinoma; H3K27me3: Trimethylation of histone H3 lysine 27; MRT: Malignant rhabdoid tumor.

\section{Acknowledgements}

Not applicable.

\section{Authors' contributions}

ZC and FJW conceived the study. FJW, YHJ, CSC, ZQC and JYJ contributed materials and methods. FJW and YHJ analyzed the data. All authors approved the final manuscript. All authors read and approved the final manuscript.

\section{Funding}

The work was supported by the National Natural Science Foundation of China (Grant No. 81930115).

\section{Availability of data and materials}

The results were analyzed online and aggregated directly from multiple databases without relevant accession numbers. Direct web links of datasets: ONCOMINE, https://www.oncomine.org/resource/login.html (Registration is needed to login into the database); Gene Expression Profiling Interactive Analysis (GEPIA), http://gepia2.cancer-pku.cn/; Kaplan-Meier Plotter, http:// kmplot.com/analysis/; The Human Protein Atlas (HPA), https://www.proteinatl as.org/; Metascape, http://metascape.org.

\section{Declarations}

Ethics approval and consent to participate Not applicable.

\section{Consent for publication}

Not applicable.

\section{Competing interests}

The authors declare no conflicts of interest in this study.

\section{Author details}

${ }^{1}$ Department of Integrative Oncology, Fudan University Shanghai Cancer Center, 270 Dong An Road, Shanghai 200032, China. ${ }^{2}$ Department of Oncology, Shanghai Medical College, Fudan University, Shanghai 200032, China.

Received: 14 March 2021 Accepted: 22 July 2021

Published online: 27 July 2021

\section{References}

1. Peterson $C L$, Dingwall A, Scott MP. Five SWI/SNF gene products are components of a large multisubunit complex required for transcriptional enhancement. Proc Natl Acad Sci USA. 1994;91(8):2905-8.

2. Shain AH, Pollack JR. The spectrum of SWI/SNF mutations, ubiquitous in human cancers. PLoS ONE. 2013;8(1):e55119.

3. Herpel E, Rieker RJ, Dienemann H, Muley T, Meister M, Hartmann A, Warth A, Agaimy A. SMARCA4 and SMARCA2 deficiency in non-small cell lung cancer: immunohistochemical survey of 316 consecutive specimens. Ann Diagn Pathol. 2017;26:47-51.

4. Chetty R, Serra S. SMARCA family of genes. J Clin Pathol. 2020;73(5):257-60.

5. Rother MB, van Attikum H. DNA repair goes hip-hop: SMARCA and CHD chromatin remodellers join the break dance. Philos Trans R Soc Lond Ser B Biol Sci. 2017;372(1731):20160285.

6. Rhodes DR, Kalyana-Sundaram S, Mahavisno V, Varambally R, Yu J, Briggs BB, Barrette TR, Anstet MJ, Kincead-Beal C, Kulkarni P, et al. Oncomine 3.0: genes, pathways, and networks in a collection of 18,000 cancer gene expression profiles. Neoplasia (New York, NY). 2007:9(2):166-80.

7. Tang Z, Li C, Kang B, Gao G, Li C, Zhang Z. GEPIA: a web server for cancer and normal gene expression profiling and interactive analyses. Nucleic Acids Res. 2017;45(W1):W98-W102.

8. Györffy B, Lanczky A, Eklund AC, Denkert C, Budczies J, Li Q, Szallasi Z. An online survival analysis tool to rapidly assess the effect of 22,277 genes on breast cancer prognosis using microarray data of 1,809 patients. Breast Cancer Res Treat. 2010;123(3):725-31.

9. Gyorffy B, Lánczky A, Szállási Z. Implementing an online tool for genome-wide validation of survival-associated biomarkers in ovariancancer using microarray data from 1287 patients. Endocr Relat Cancer. 2012;19(2):197-208

10. Szász AM, Lánczky A, Nagy Á, Förster S, Hark K, Green JE, Boussioutas A, Busuttil R, Szabó A, Győrffy B. Cross-validation of survival associated biomarkers in gastric cancer using transcriptomic data of 1,065 patients. Oncotarget. 2016;7(31):49322-33.

11. Asplund A, Edqvist PH, Schwenk JM, Pontén F. Antibodies for profiling the human proteome-The Human Protein Atlas as a resource for cancer research. Proteomics. 2012;12(13):2067-77.

12. Zhou Q, Zhang F, He Z, Zuo M-Z. E2F2/5/8 serve as potential prognostic biomarkers and targets for human ovarian cancer. Front Oncol. 2019;9:161.

13. Buchholz M, Braun M, Heidenblut A, Kestler HA, Klöppel G, Schmiegel W, Hahn SA, Lüttges J, Gress TM. Transcriptome analysis of microdissected pancreatic intraepithelial neoplastic lesions. Oncogene. 2005;24(44):6626-36.

14. Logsdon CD, Simeone DM, Binkley C, Arumugam T, Greenson JK, Giordano TJ, Misek DE, Kuick R, Hanash S. Molecular profiling of pancreatic adenocarcinoma and chronic pancreatitis identifies multiple genes differentially regulated in pancreatic cancer. Can Res. 2003;63(10):2649-57.

15. Grützmann R, Pilarsky C, Ammerpohl O, Lüttges J, Böhme A, Sipos B, Foerder M, Alldinger I, Jahnke B, Schackert HK, et al. Gene expression profiling of microdissected pancreatic ductal carcinomas using high-density DNA microarrays. Neoplasia (New York, NY). 2004;6(5):611-22.

16. Pei H, Li L, Fridley BL, Jenkins GD, Kalari KR, Lingle W, Petersen G, Lou Z, Wang $L$. FKBP51 affects cancer cell response to chemotherapy by negatively regulating Akt. Cancer Cell. 2009;16(3):259-66.

17. Takeshima H, Niwa T, Takahashi T, Wakabayashi M, Yamashita S, Ando T, Inagawa $Y$, Taniguchi $\mathrm{H}$, Katai H, Sugiyama T, et al. Frequent involvement of chromatin remodeler alterations in gastric field cancerization. Cancer Lett. 2015;357(1):328-38. 
18. Zhang Z, Wang F, Du C, Guo H, Ma L, Liu X, Kornmann M, Tian X, Yang Y. BRM/SMARCA2 promotes the proliferation and chemoresistance of pancreatic cancer cells by targeting JAK2/STAT3 signaling. Cancer Lett. 2017:402:213-24.

19. Wilson BG, Helming KC, Wang X, Kim Y, Vazquez F, Jagani Z, Hahn WC, Roberts CW. Residual complexes containing SMARCA2 (BRM) underlie the oncogenic drive of SMARCA4 (BRG1) mutation. Mol Cell Biol. 2014;34(6):1136-44.

20. Pasic I, Wong KM, Lee JJ, Espin-Garcia O, Brhane Y, Cheng D, Chen Z, Patel D, Brown C, Bucur R, et al. Two BRM promoter polymorphisms predict poor survival in patients with hepatocellular carcinoma. Mol Carcinog. 2018;57(1):106-13.

21. Wu J, He K, Zhang Y, Song J, Shi Z, Chen W, Shao Y. Inactivation of SMARCA2 by promoter hypermethylation drives lung cancer development. Gene. 2019;687:193-9.

22. Yamamichi N, Inada K, Ichinose M, Yamamichi-Nishina M, Mizutani T, Watanabe H, Shiogama K, Fujishiro M, Okazaki T, Yahagi N, et al. Frequent loss of Brm expression in gastric cancer correlates with histologic features and differentiation state. Can Res. 2007;67(22):10727-35.

23. Xu X, Zheng Z, Jia L, Suo S, Liu B, Shao T, Tu Q, Hua Y, Xu H. Overexpression of SMARCA2 or CAMK2D is associated with cisplatin resistance in human epithelial ovarian cancer. Oncol Lett. 2018;16(3):3796-804.

24. Liu L, Liu H, Zhou Y, He J, Liu Q, Wang J, Zeng M, Yuan D, Tan F, Zhou Y, et al. HLTF suppresses the migration and invasion of colorectal cancer cells via TGF- $\beta$ /SMAD signaling in vitro. Int J Oncol. 2018;53(6):2780-8.

25. Adra CN, Donato JL, Badovinac R, Syed F, Kheraj R, Cai H, Moran C, Kolker MT, Turner H, Weremowicz S, et al. SMARCAD1, a novel human helicase family-defining member associated with genetic instability: cloning, expression, and mapping to 4q22-q23, a band rich in breakpoints and deletion mutants involved in several human diseases. Genomics. 2000;69(2):162-73.

26. Arafat K, Al Kubaisy E, Sulaiman S, Karam SM, Al Natour Z, Hassan AH, Attoub S. SMARCAD1 in breast cancer progression. Cell Physiol Biochem. 2018;50(2):489-500

27. Al Kubaisy E, Arafat K, De Wever O, Hassan AH, Attoub S. SMARCAD1 knockdown uncovers its role in breast cancer cell migration, invasion, and metastasis. Expert Opin Ther Targets. 2016;20(9):1035-43.

28. Tapak L, Saidijam M, Sadeghifar M, Poorolajal J, Mahjub H. Competing risks data analysis with high-dimensional covariates: an application in bladder cancer. Genom Proteom Bioinform. 2015:13(3):169-76.

29. Liu F, Xia Z, Zhang M, Ding J, Feng Y, Wu J, Dong Y, Gao W, Han Z, Liu Y, et al. SMARCAD1 promotes pancreatic cancer cell growth and metastasis through Wht/ $\beta$-catenin-mediated EMT. Int J Biol Sci. 2019;15(3):636-46.

30. Vangamudi B, Paul TA, Shah PK, Kost-Alimova M, Nottebaum L, Shi X, Zhan Y, Leo E, Mahadeshwar HS, Protopopov A, et al. The SMARCA2/4 ATPase domain surpasses the bromodomain as a drug target in SWI/
SNF-mutant cancers: insights from cDNA rescue and PFI-3 inhibitor studies. Can Res. 2015;75(18):3865-78.

31. Lissanu Deribe Y, Sun Y, Terranova C, Khan F, Martinez-Ledesma J, Gay J, Gao G, Mullinax RA, Khor T, Feng N, et al. Mutations in the SWI/SNF complex induce a targetable dependence on oxidative phosphorylation in lung cancer. Nat Med. 2018;24(7):1047-57.

32. The Cancer Genome Altas Research N. Comprehensive molecular profiling of lung adenocarcinoma. Nature 2014;511(7511):543-50.

33. Jelinic P, Mueller JJ, Olvera N, Dao F, Scott SN, Shah R, Gao J, Schultz N, Gonen M, Soslow RA, et al. Recurrent SMARCA4 mutations in small cell carcinoma of the ovary. Nat Genet. 2014;46(5):424-6.

34. Jin Q, Mao X, Li B, Guan S, Yao F, Jin F. Overexpression of SMARCA5 correlates with cell proliferation and migration in breast cancer. Tumour Biol. 2015;36(3):1895-902.

35. Wang H, Tan L, Dong X, Liu L, Jiang Q, Li H, Shi J, Yang X, Dai X, Qian Z, et al. MiR-146b-5p suppresses the malignancy of GSC/MSC fusion cells by targeting SMARCA5. Aging. 2020;12(13):13647-67.

36. Puccetti MV, Adams CM, Kushinsky S, Eischen CM. Smarcal1 and Zranb3 protect replication forks from Myc-induced DNA replication stress. Can Res. 2019;79(7):1612-23.

37. Law CT, Wei L, Tsang FH, Chan CY, Xu IM, Lai RK, Ho DW, Lee JM, Wong CC, Ng IO, et al. HELLS regulates chromatin remodeling and epigenetic silencing of multiple tumor suppressor genes in human hepatocellular carcinoma. Hepatology (Baltimore, MD). 2019;69(5):2013-30.

38. Zhang G, Dong Z, Prager BC, Kim $\sqcup$, Wu Q, Gimple RC, Wang X, Bao S, Hamerlik P, Rich JN. Chromatin remodeler HELLS maintains glioma stem cells through E2F3 and MYC. JCl Insight. 2019;4(7): e126140.

39. Wilson BG, Roberts CW. SWI/SNF nucleosome remodellers and cancer. Nat Rev Cancer. 2011;11(7):481-92.

40. Finetti MA, Grabovska Y, Bailey S, Williamson D. Translational genomics of malignant rhabdoid tumours: current impact and future possibilities. Semin Cancer Biol. 2020;61:30-41.

41. Gounder M, Schöffski P, Jones RL, Agulnik M, Cote GM, Villalobos VM, Attia S, Chugh R, Chen TW, Jahan T, et al. Tazemetostat in advanced epithelioid sarcoma with loss of INI1/SMARCB1: an international, open-label, phase 2 basket study. Lancet Oncol. 2020;21(11):1423-32.

42. Ma A, Stratikopoulos E, Park KS, Wei J, Martin TC, Yang X, Schwarz M, Leshchenko V, Rialdi A, Dale B, et al. Discovery of a first-in-class EZH2 selective degrader. Nat Chem Biol. 2020;16(2):214-22.

\section{Publisher's Note}

Springer Nature remains neutral with regard to jurisdictional claims in published maps and institutional affiliations.
Ready to submit your research? Choose BMC and benefit from:

- fast, convenient online submission

- thorough peer review by experienced researchers in your field

- rapid publication on acceptance

- support for research data, including large and complex data types

- gold Open Access which fosters wider collaboration and increased citations

- maximum visibility for your research: over $100 \mathrm{M}$ website views per year

At BMC, research is always in progress.

Learn more biomedcentral.com/submissions 